\title{
Inelastic neutron scattering study of methyl groups rotation in some methylxanthines
}

M. Prager, A. Pawlukojc, A. Wischnewski, and J. Wuttke

Citation: The Journal of Chemical Physics 127, 214509 (2007); doi: 10.1063/1.2803187

View online: https://doi.org/10.1063/1.2803187

View Table of Contents: http://aip.scitation.org/toc/jcp/127/21

Published by the American Institute of Physics

\section{Articles you may be interested in}

SPHERES, Jülich's high-flux neutron backscattering spectrometer at FRM II

Review of Scientific Instruments 83, 075109 (2012); 10.1063/1.4732806

The new cold neutron chopper spectrometer at the Spallation Neutron Source: Design and performance Review of Scientific Instruments 82, 085108 (2011); 10.1063/1.3626935

The cold neutron chopper spectrometer at the Spallation Neutron Source-A review of the first 8 years of operation

Review of Scientific Instruments 87, 093902 (2016); 10.1063/1.4962024

A sample holder for simultaneous Raman and neutron vibrational spectroscopy

Review of Scientific Instruments 89, 013112 (2018); 10.1063/1.4997933

A time-of-flight backscattering spectrometer at the Spallation Neutron Source, BASIS

Review of Scientific Instruments 82, 085109 (2011); 10.1063/1.3626214

Distributions of methyl group rotational barriers in polycrystalline organic solids

The Journal of Chemical Physics 139, 204501 (2013); 10.1063/1.4830411

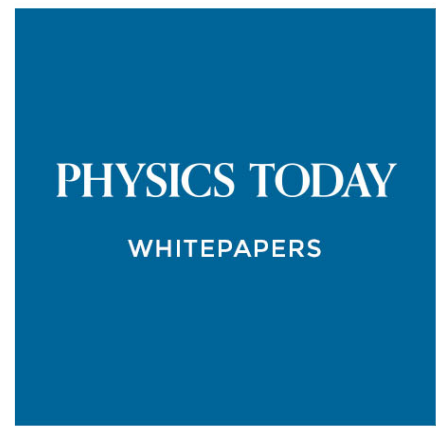

ADVANCED LIGHT CURE ADHESIVES

Take a closer look at what these environmentally friendly adhesive systems can do

\section{READ NOW}

PRESENTED BY Q8 MASTERBOND 


\title{
Inelastic neutron scattering study of methyl groups rotation in some methylxanthines
}

\author{
M. Prager ${ }^{\mathrm{a})}$ \\ Institut für Festkörperforschung, Forschungszentrum Jülich, D-52425 Jülich, Germany \\ and Jülich Centre for Neutron Science, Lichtenbergstrasse 4, D-85747 Garching, Germany
}

\author{
A. Pawlukojc \\ Institut of Nuclear Chemistry and Technology, Warsaw 03-195, Poland
}

A. Wischnewski

Institut für Festkörperforschung, Forschungszentrum Jülich, D-52425 Jülich, Germany

J. Wuttke

Jülich Centre for Neutron Science, Lichtenbergstrasse 4, D-85747 Garching, Germany

(Received 13 August 2007; accepted 5 October 2007; published online 7 December 2007)

\begin{abstract}
The three isomeric dimethylxanthines and trimethylxanthine are studied by neutron spectroscopy up to energy transfers of $100 \mathrm{meV}$ at energy resolutions ranging from $0.7 \mu \mathrm{eV}$ to some meV. The loss of elastic intensity with increasing temperature can be modeled by quasielastic methyl rotation. The number of inequivalent methyl groups is in agreement with those of the room temperature crystal structures. Activation energies are obtained. In the case of theophylline, a doublet tunneling band is observed at 15.1 and $17.5 \mu \mathrm{eV}$. In theobromine, a single tunneling band at $0.3 \mu \mathrm{eV}$ is found. Orientational disorder in caffeine leads to a $2.7 \mu \mathrm{eV}$ broad distribution of tunneling bands around the elastic line. At the same time, broad low energy phonon spectra characterize an orientational glassy state with weak methyl rotational potentials. Librational energies of the dimethylxanthines are clearly seen in the phonon densities of states. Rotational potentials can be derived which explain consistently all observables. While their symmetry in general is threefold, theophylline shows a close to sixfold potential reflecting a mirror symmetry. (C) 2007 American Institute of Physics.
\end{abstract}

[DOI: $10.1063 / 1.2803187]$

\section{INTRODUCTION}

The investigations of biologically active materials as the drugs paracetamol ${ }^{1}$ or aspirin ${ }^{2}$ have given insight in chemical and dynamical properties which might also be of importance in their biological function. In the case of paracetamol, a sufficiently correct modeling with modern lattice dynamical programs was only possible after allowing polarization or charge transfer between molecules due to hydrogen bonds. In one component systems, the effect of hydrogen bonds leads to a charge redistribution within a molecule due to the influence of an environment. Mathematical modeling of the lattice dynamics is based on the precise knowledge of the three dimensional crystal structure of the molecular crystal. Methyl group rotation represents the most sensitive probe of the intermolecular interaction. It is especially informative in systems with hydrogen bonds.

The methylxanthines with two and three methyl groups represent a biologically important class of molecules. These rather simple double ring molecules include the natural drugs caffeine, theobromine, and theophylline. The molecules are shown in Fig. 1.

Caffeine $\mathrm{C}_{8} \mathrm{H}_{10} \mathrm{~N}_{4} \mathrm{O}_{2}$ is a xanthine alkaloid compound that acts as a stimulant in humans. It is found in the leaves and beans of the coffee plant, in tea, yerba mate, and guarana

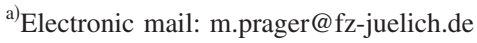

berries, the kola nut, the Yaupon Holly, and in small quantities in cocoa. Depending on the origin, the molecule can also be named guaranine, mateine, or theine. Overall, caffeine is found in the beans, leaves, and fruits of over 60 plants, where it acts as a natural pesticide that paralyzes and kills
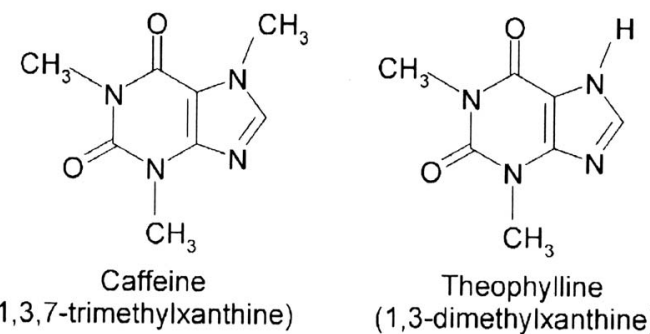

Theophylline (1,3-dimethylxanthine)<smiles>Cn1cnc2c1c(=O)[nH]c(=O)n2C</smiles>

Theobromine (3,7-dimethylxanthine)

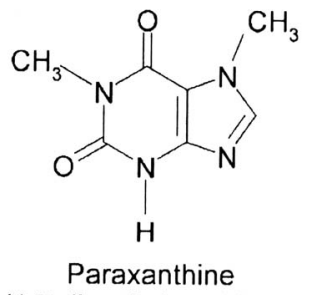

(1,7-dimethylxanthine)

FIG. 1. Molecular structures of caffeine, theophylline, theobromine, and paraxanthine. 
certain insects feeding on the plants. Caffeine is a central nervous system stimulant, having the effect of temporarily warding off drowsiness and restoring alertness. Beverages containing caffeine, such as coffee, tea, soft drinks, and energy drinks, enjoy great popularity; caffeine is the world's most widely consumed psychoactive substance, but unlike most other psychoactive substances, it is legal and unregulated in nearly all jurisdictions. By oxidative demethylation, caffeine is metabolized in the liver to the dimethylxanthines theobromine, theophylline, and paraxanthine. In a second step, demethylation leads to monomethylxanthines.

Theophylline is a drug, a phosphodiesterase inhibitor, which is widely used in therapy for respiratory diseases. It is naturally found in black tea and green tea. The main actions of theophylline are relaxation of bronchial smooth muscle and positive inotropic (increases heart muscle contractibility and efficiency) and positive chronotropic (increases heart rate) impact. It lowers blood pressure, increases renal blood flow, and has some anti-inflammatory effect. The therapeutic use of theophylline focuses on reducing chronic obstructive diseases of the airways, chronic obstructive pulmonary disease, and bronchial asthma.

Theobromine is an alkaloid found in cocoa and chocolate, in doses which are safe for humans to consume in large quantities. It is known to induce mutations in bacteria and lower eukaryotes but does not seem to cause mutations in higher eukaryotes, such as humans. Theobromine has an antitussive effect superior to codeine by suppressing vagus nerve activity.

Paraxanthine is not produced by plants and is only observed in nature as a metabolite of caffeine in animals. The compound is produced from caffeine (1,3,7-trimethylxanthine) breakdown. After intake, roughly $84 \%$ of caffeine is demethylated at the 3-position to yield paraxanthine. Paraxanthine has a number of physiological effects on animals. In humans, the compound acts as a nonselective, competitive inhibitor of adenosine receptors. ${ }^{3,4}$ As a result, paraxanthine triggers an elevated diastolic blood pressure and an increase in plasma epinephrine levels. Furthermore, the compound is responsible for the lipolytic properties of caffeine, and its presence in the blood causes no increase in serum free fatty acid concentration.

The crystal structures of anhydrous theophylline, ${ }^{5,6}$ theobromine, ${ }^{7}$ and paraxanthine ${ }^{8}$ are determined at room temperature. Their space groups are orthorhombic Pna $2_{1}$, monoclinic $P 2_{1} / c$, and monoclinic $P 2_{1} / n$, respectively. In the asymmetric crystallographic unit cell, theophylline and theobromine crystals have one and paraxanthine crystals has two molecules. This means that the number of inequivalent methyl groups for theophylline and theobromine is equal two, for paraxanthine, equal four. More complicated is the case of caffeine. Crystallographic investigations on powder anhydrous caffeine show that at $278 \mathrm{~K}$, caffeine crystallizes in the trigonal $R 3 c$ space group with six molecules in the crystallographic unit cell. ${ }^{9,10}$ At this temperature, the molecules are orientationally dynamically disordered in a socalled plastic phase, where a molecule at every site can adopt three preferential orientations with equal occupation probability. Below $250 \mathrm{~K}$, the dynamical disorder transforms into a glassy crystal state.

The methylxanthines show a remarkable similarity to the nuleic acids. The six-membered ring including the two double bound oxygens reappears in thymine and uracil and, with an $\mathrm{NH}_{2}$ group replacing one oxygen, in cytosine. The full double ring structure is the same as in guanine and adenine. The most prominent property of nucleic acids is the selective hydrogen bonding between each two pairs which is the fundament of the genetic code. Methylxanthines form similar hydrogen bonded networks in the neat material. ${ }^{7}$ The formation of a hydrogen bonded stoichiometric molecular alloy with a bioreceptor ${ }^{11}$ contributes to an understanding of its interaction with small molecules and the resulting antibacterial activity of caffeine.

Special features of methylxanthines are the different binding sites of methyl groups. It is well-known from mixed halomesitylenes ${ }^{12}$ that the hindering potential of methyl groups can strongly and often unexpectedly change compared to pure compounds with their position and the neighborhood in the ring. Since rotational potentials of halomesitylenes are sufficiently small, rotational tunneling spectroscopy can complement and improve the information extracted from vibrational spectra. Especially, the assignment of soft methyl librations gets more convincing with the observation of corresponding tunneling bands.

In this paper, we investigate dynamical properties of caffeine, theobromine, theophylline, and 1,7-dimethylxanthine in the regime of lattice modes using inelastic neutron scattering (INS) techniques. Special attention shall be given to the rotational dynamics of methyl groups. INS spectroscopy is best suited with this respect because librational modes are not hidden by selection rules like in optical spectroscopy and appear as strong bands due to the large scattering cross section of hydrogen. The low energy region (up to $80 \mathrm{meV}$ ) in which torsional vibrations of the methyl groups are expected can similarly be measured well with direct and inverse thermal time-of-flight spectrometers.

\section{THEORY}

The standard description of methyl rotational tunneling and quasielastic scattering is the single particle model. ${ }^{13}$ In this mean field theory, the environment of the molecule is represented by a rotational potential $V(\varphi)$ of the threefold symmetry of the methyl group,

$$
V(\varphi)=\sum_{j=1}^{J} \frac{V_{3 j}}{2}\left(1-\cos \left(3 j \varphi-\varphi_{3 j}\right)\right) .
$$

The expansion is, in general, limited to $J=2$. In a static environment, i.e., at low sample temperatures, this potential characterizes a hindered quantum rotor with eigenenergies $E_{i}$ given by the stationary single particle Schrödinger equation, ${ }^{13,14}$ 


$$
\left\{-B \frac{\partial^{2}}{\partial \varphi^{2}}+V(\varphi)\right\} \Psi_{i}=E_{i} \Psi_{i} .
$$

$B=\hbar^{2} / 2 \Theta=0.655 \mathrm{meV}$ is the rotational constant of the methyl group with the moment of inertia $\Theta$. With increasing potential strength the free rotor levels get arranged similar to a harmonic oscillator with split librational levels. The splitting is due to the overlap of wave functions in the different pockets. It is called tunnel splitting and is of the order of $\mu \mathrm{eV}$, while the separation of the two lowest pairs, the fundamental librational energy $E_{01}$, is of the order of meV.

High resolution neutron spectroscopy is an especially successful technique to observe the ground state tunnel splitting $\hbar \omega_{t}$ of weakly hindered rotors. The simple scattering function of a single methyl rotor $^{13,14}$ becomes more complex for xanthines containing a number $N$ of inequivalent methyl groups with $N^{\prime} \leqslant N$ tunnel splittings resolved. In addition, a number $M$ of individual other protons exist which scatter only elastically. The scattering function of such a system is

$$
\begin{aligned}
S(Q, \omega)= & \left(N^{\prime}\left(1+2 j_{0}(Q d)\right)+3\left(N-N^{\prime}\right)+M\right) \delta(\omega) \\
& +\sum_{n=1}^{N^{\prime}}\left(\frac{2}{3}-\frac{2}{3} j_{0}(Q d)\right)\left\{L(\omega, \Gamma)+L\left(\omega+\omega_{t n}, \Gamma\right)\right. \\
& \left.+L\left(\omega-\omega_{t n}, \Gamma\right)\right\}
\end{aligned}
$$

with momentum transfer $Q$ and distance $d$ of the protons within the methyl group. $L$ stands for a Lorentzian function characteristic of a stochastic dynamical process with a single time constant.

At low temperature when the linewidth is much smaller than the instrumental energy resolution, $\Gamma \ll \delta E_{\text {res }}$, the Lorentzian degenerates to a $\delta$ function. Equation (3) yields then a ratio of inelastic transition to the total elastic intensity,

$$
\frac{I_{\text {inel }}(n)}{I_{\mathrm{el}}}=\frac{2-2 j_{0}}{N^{\prime}\left(5+4 j_{0}\right)+9\left(N-N^{\prime}\right)+3 M} .
$$

$N, N^{\prime}$, and $M$ are characteristic of the compound. This relation allows us to extract $N^{\prime}$ by comparing elastic and tunneling intensities. Thus, conclusions about occurrence probabilities and therefrom molecular site symmetries become possible. It must be kept in mind, however, that elastic scattering from other sources, i.e., other nuclei, coherent (Bragg) scattering, etc., must be removed from $I_{\mathrm{el}}$ before evaluating Eq. (4).

At a higher temperature, jump reorientation of methyl groups leads to quasielastic scattering,

$$
\begin{aligned}
S(Q, \omega)= & \left(N^{\prime}\left(1+2 j_{0}(Q d)\right)+3\left(N-N^{\prime}\right)+M\right) \delta(\omega) \\
& +\sum_{n=1}^{N^{\prime}} 2\left(1-j_{0}(Q d)\right) L\left(\omega, \Gamma_{n}\right)
\end{aligned}
$$

with a Lorentzian linewidth $\Gamma_{n}$. Thermally activated dynamical processes follow, in general, an Arrhenius dependence,

$$
\Gamma_{n}(T)=\Gamma_{0 n} \exp \left(-E_{a}(n) / k T\right),
$$

with two free parameters $E_{a}(n)$ and $\Gamma_{0 n}$. For methyl group rotation, $\Gamma_{0 n}$ is of the order of librational energies and depends little on the rotational barrier, etc. ${ }^{15}$
A first estimate of the activation energy of methyl group rotation (or any other stochastic dynamical process) can be obtained by the fixed-window technique. Instead of taking spectra the elastic intensity integrated with the energy resolution of the used instrument is studied as a function of sample temperature. Following Ref. 16 the elastic intensity obeys a temperature dependence,

$$
\begin{aligned}
I(Q, T) \sim & \operatorname{DWF}(Q) \cdot\{\operatorname{EISF}(Q) \\
& \left.-(1-\operatorname{EISF}(Q)) *\left(\frac{2}{\pi} \operatorname{atan}\left(\frac{\delta E_{\text {res }}}{\Gamma_{n}(T)}\right)\right)\right\} .
\end{aligned}
$$

A Debye-Waller factor $\operatorname{DWF}(Q)$ characterizes the continuous decrease of elastic intensity with temperature. The elastic incoherent structure factor $\operatorname{EISF}(Q)$ determines the height of the steplike intensity loss due to a specific quasielastic process. The temperature dependence comes in via the Arrhenius dependence of $\Gamma$ [Eq. (6)]. We can define a temperature $T_{1 / 2}$ where half of the quasielastic intensity will have left this elastic window. At this temperature the Lorentzian linewidth $\Gamma_{n}\left(T_{1 / 2}\right)$ becomes equal to the energy resolution $\delta E_{\text {res }}$. If $\Gamma_{0}$ is roughly known, one can guess an activation energy of methyl group rotation just from $T_{1 / 2}$. Furthermore we can define a temperature range $\Delta T$ in which most quasielastic intensity leaves the elastic window. Arbitrarily we define this range by temperatures where $\Gamma_{n}$ changes from $0.5 \delta E_{\text {res }}$ to $2 \delta E_{\text {res }}$. For $\delta E_{\text {res }}=1 \mu \mathrm{eV}$ and an average experimental value $\Gamma_{0}=8000 \mu \mathrm{eV}$, we arrive at simple thumb rules as follows:

$$
\begin{aligned}
& \frac{E_{a}}{T_{1 / 2}}=9.0 \\
& \frac{\Delta T}{T_{1 / 2}}=\frac{\ln 4}{\frac{E_{a}}{T_{1 / 2}}}=0.15 .
\end{aligned}
$$

The value of 0.15 will slightly change with the limits chosen; however, the constant relation is maintained. As an application of the second equation a decomposition of fixed-window scans into subbands becomes possible. If the experiment yields a temperature range clearly larger than the characteristic constant of 0.15 , then the molecular crystal studied may either contain inequivalent overlapping rotor systems or significant disorder with a broad range of rotational potentials.

One of the problems with this analysis is that the elastic intensity from atoms not involved in the quasielastic dynamics or Bragg intensity is superimposed to the incoherent scattering of Eq. (7). In complex structures, Bragg intensities also change with temperature and cannot be removed in a simple way. The clearest information is contained and should, therefore, be extracted from detectors little or not disturbed by Bragg reflections.

\section{EXPERIMENTAL}

Samples were supplied by the Fluka company and are used as obtained. Flat Al containers were used. Effective sample thickness was $0.5 \mathrm{~mm}$ and the samples were oriented 


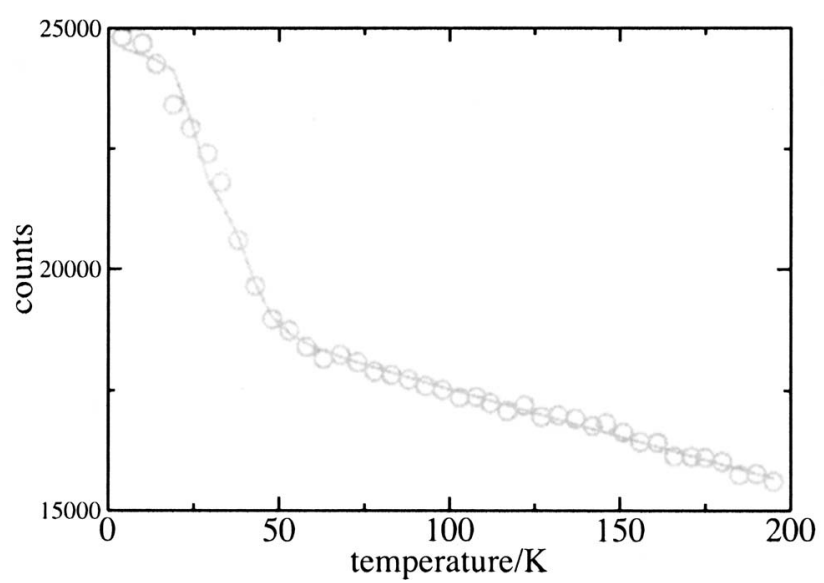

FIG. 2. Fixed-window scan of theophylline. $Q=1.72 \AA^{-1}$.

under $45^{\circ}$ to the beam. This leads to scattering probabilities around $15 \%$ and allows no neglect multiple scattering effects.

Some preliminary high resolution inelastic spectra and the elastic fixed-window scans were measured at the neutron backscattering spectrometer BSS of Forschungszentrum Jülich $^{17}$ with unpolished $\mathrm{Si}[111]$ analyzer crystals. Two monochromators were used. Unpolished $\mathrm{Si}[111]$ gives access to an energy range of $\pm 18 \mu \mathrm{eV}$ at an energy resolution $\delta E_{\text {res }}=0.99 \mu \mathrm{eV}$. With the $\mathrm{SiGe}[111]$ offset monochromator the accessible energy range is shifted to $-32 \mu \mathrm{eV} \leqslant \delta E$ $\leqslant 4 \mu \mathrm{eV}$ at a reduced energy resolution $\delta E_{\text {res }}=1.7 \mu \mathrm{eV}$. The angular resolutions of $19^{\circ}$ for large scattering angles and of $9^{\circ}$ for the small ones allows us to determine the $Q$ dependence of the elastic incoherent structure factor EISF.

Another set of spectra was measured with the new backscattering spectrometer SPHERES of the Jülich Center for Neutron Science (JCNS) at the research reactor FRM2, Munich. ${ }^{18}$ This instrument has a higher flux, better energy resolution of $0.74 \mu \mathrm{eV}$ with the setup with unpolished Si[111] monochromator and analyzer crystals, and a larger dynamics range of up to $\sim \pm 30 \mu \mathrm{eV}$ than the former BSS instrument.

Lattice and internal modes up to an energy transfer of $\sim 50 \mathrm{meV}$ were measured at the thermal time-of-flight spec-

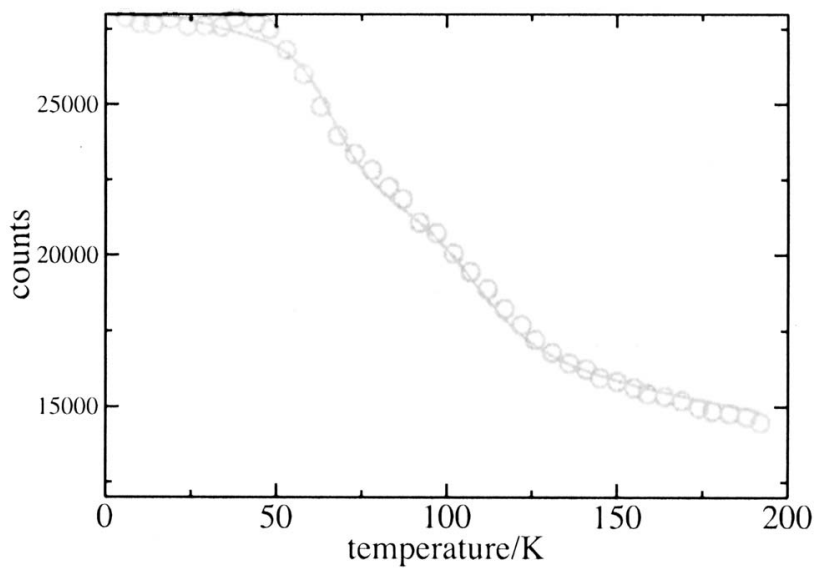

FIG. 3. Fixed-window scan of theobromine. $Q=1.72 \AA^{-1}$.

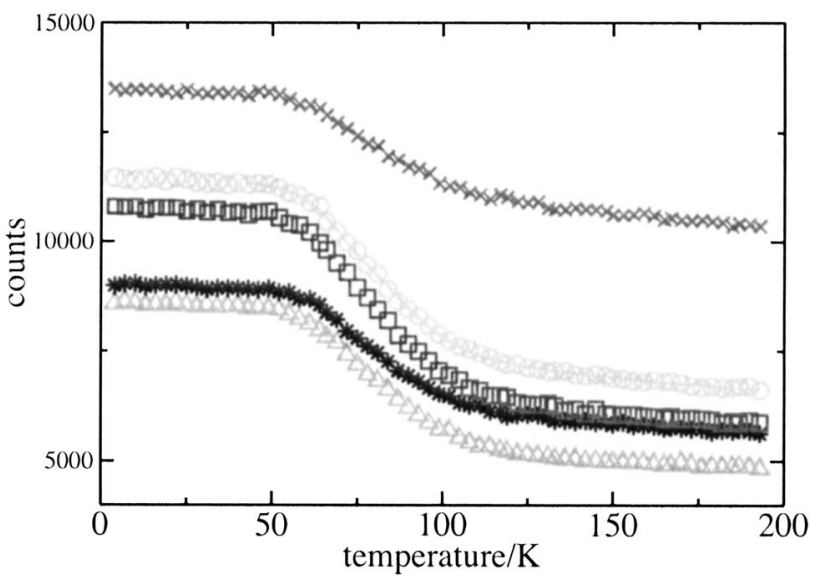

FIG. 4. Fixed-window scan of 1,7-dimethylxanthine. Momentum transfers $Q=1.87(\square), 1.72(\bigcirc), 1.40(\triangle), 1.29\left(^{*}\right)$, and $1.02(\mathrm{x}) \AA^{-1}$.

trometer SV29 (Ref. 17) with energy resolutions $\delta E_{\text {res }}=2$ and $5 \mathrm{meV}$ using wavelengths $\lambda=1.76$ and $1.17 \AA$, respectively. For caffeine the inverse time-of-flight spectrometer NERA (Ref. 19) of the Joint Institute of Nuclear Research (JINR) in Dubna, Russia was used.

\section{RESULTS AND DISCUSSION}

\section{A. Fixed-window scans}

An overview on methyl group dynamics and the heights of rotational barriers is obtained from fixed-window scans. The raw data are shown in Figs. 2-5. The full potential of the fixed-window technique is, for example, shown in Ref. 20. In our materials, superposition of scattering from inequivalent rotors introduces too many free parameters for a similar analysis. We focus our interest on the EISF and a rough estimate of activation energies.

For all materials, the intensity loss with temperature, 1-EISF, increases continuously with momentum transfer, as shown typically in Fig. 4. This means that a local motion is the responsible process. For the proton-proton distance in the methyl groups the minimum (maximum) of the (in)elastic structure factor would appear at $2.7 \AA^{-1}$. This value is outside the range of the BSS spectrometer. The continuous in-

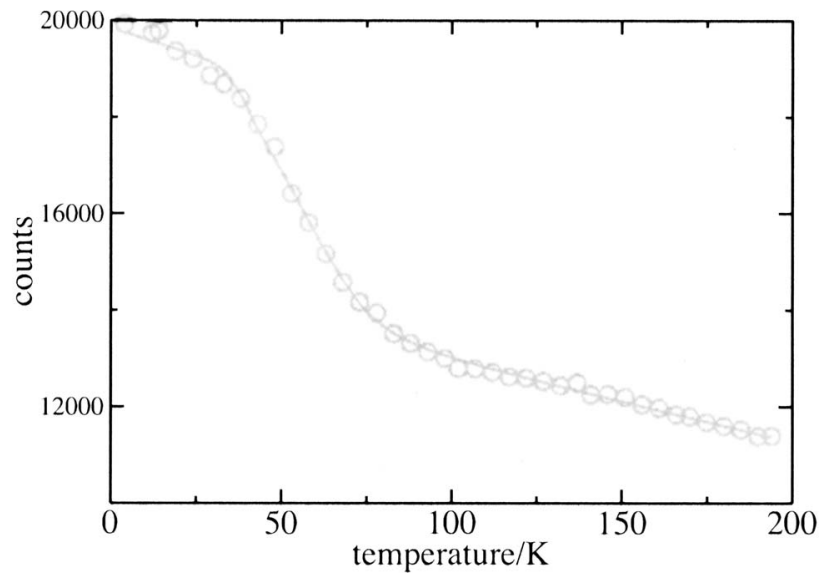

FIG. 5. Fixed-window scan of caffeine. $Q=1.56 \AA^{-1}$. 
TABLE I. Characteristic parameters of theophylline, theobromine, caffeine, and paraxanthine extracted from fixed-window scans. Momentum transfer is $Q$.

\begin{tabular}{lrcccc}
\hline \hline Sample & $\begin{array}{c}T_{1 / 2} \\
(\mathrm{~K})\end{array}$ & $\operatorname{EISF}(Q)$ & Model & $\begin{array}{c}E_{a} \\
(\mathrm{meV})\end{array}$ & $\Delta T / T_{1 / 2}$ \\
\hline Theophylline & 24 & & 2 tun & $(19)$ & \\
& 41 & $0.53\left(1.72 \AA^{-1}\right)$ & & $(32)$ & \\
Theobromine & 68 & $0.56\left(1.72 \AA^{-1}\right)$ & 2 class & 53 & 0.17 \\
& 113 & & & 88 & 0.26 \\
Caffeine & 55 & $0.59\left(1.56 \AA^{-1}\right)$ & 2 tun+1 class & 43 & 0.35 \\
Paraxanthine & 82 & $0.68\left(1.72 \AA^{-1}\right)$ & $2(4)$ class & 64 & 0.31 \\
\hline \hline
\end{tabular}

crease of the lost intensity with scattering angle is qualitatively consistent with the EISF of methyl group rotation.

The presence of inequivalent methyl groups requires that the intensity profile versus temperature must be fitted by a sum of expressions [Eq. (7)], plus an arbitrary elastic intensity due to other sources of incoherent elastic and Bragg scatterings. A fit with parameters presented in columns 3 and 5 of Table I is shown for a selected scattering angle of each sample as solid lines in the figures. Column 2 shows $T_{1 / 2}$ as derived from Eq. (8). Column 4 characterizes the model used. "Class" means that the low temperature intensity is given by Eq. (5), while "tun" means that the same functional dependence $I(Q, T)$ is used, but the step height is reduced, since the tunneling bands at low temperature are outside the elastic line [Eq. (3)]. In such a case, the meaning of $E_{a}$ is also modified. This is indicated by brackets in Table I.

At first, we deal with the number of methyl groups involved in the dynamics. For this purpose the intensity at high temperature has to be corrected for the incoherent background of the protons not contained in methyl groups and much smaller incoherent scattering from nonhydrogenous atoms. Secondly, Bragg scattering to the elastic intensity has to be removed. While the first effect is isotropic and almost quantitatively known, essentially due to two framework protons for the dimethylxanthines and one for caffeine, the latter is unknown but may be important due to the large coherent scattering cross section of nitrogen. For the quantitative analysis we assume that the detector with minimum elastic intensity at still reasonably large momentum transfer is not contaminated by coherent elastic scattering. These are detectors at scattering angles of $100^{\circ}$ and $118^{\circ}$. To remove the influence of the Debye-Waller factor we extrapolate the intensity measured at $T=200 \mathrm{~K}$ down to $4 \mathrm{~K}$ along the slope of the curves. If we call this value $I^{\prime}(200 \mathrm{~K})$, then $I^{\prime}(200 \mathrm{~K}) / I(4 \mathrm{~K})$ can be called the EISF and is determined here by the scattering function [Eq. (5)]. The EISFs shown in column 3 of Table I are obtained for the given momentum transfer. Due to the various corrections applied the experimental EISF values are affected with error bars of the order of $20 \%$. Comparing these values with those calculated for combinations of scattering functions [Eqs. (3) and (5)] shows that all methyl groups participate in the quasielastic scattering.

Next, we combine the fixed-window results with the knowledge from the crystal structure and try to characterize dynamically the inequivalent methyl species. For theobromine the loss of intensity occurs in two steps of equal height. The steps appear at rather high temperature and can be unambiguously assigned to classical stochastic jumps of inequivalent methyl groups of equal crystallographic occurrence probabilities across two clearly distinguishable rotational barriers. The sharpness of the steps, characterized by the last row of Table I, shows that both barriers are welldefined.

In the case of theophylline, the intensity loss appears at temperatures below $\sim 40 \mathrm{~K}$. At such low temperatures quantum effects modify the temperature dependence predicted from classical jump reorientation. Due to the large tunnel splitting the larger part of the quasielastic intensity, as defined by Eq. (5), is outside the elastic window at the lowest temperature already. This reduces the height of the step. In detail, ${ }^{14}$ the intensity lost by broadening $E_{a}-E_{b}$ transitions is partly compensated by $A-E$ transitions shifting toward the elastic line while broadening at the same time. At this temperature the prefactor and the meaning of the slope in an Arrhenius modeling are not clear. ${ }^{21}$ Precise answers may only be obtained from high resolution quasielastic experiments. ${ }^{21,22}$

The width of the single step in paraxanthine is significantly broader (column 6 of Table I) than expected for a single dynamical process. Since all methyl groups rotate, we must conclude that the four inequivalent species feel rather similar rotational potentials. The corresponding values of $T_{1 / 2}$ are too close to allow for an independent fitting of four species. Therefore, the solid line in Fig. 4 used two inequivalent $\mathrm{CH}_{3}$ groups only with doubled occurrence probabilities.

In the orientational glass state of caffeine, every methyl group experiences a different rotational potential. A large width of the corresponding potential distribution function leads to significantly broadened temperature interval $\Delta T$, where the intensity leaves the elastic window. Furthermore, the low temperatures where the elastic intensity gets lost indicate the presence of resolved tunneling species related to the lower potentials of the distribution. The overlap of quantum and classical behavior leads to the broadest step width in this material (column 6 of Table I).

\section{B. Rotational tunneling}

\section{Paraxanthine}

The spectrum from paraxanthine can be described by a resolution function. As a resolution function the elastic part of the theophylline spectrum is used. Identical sample geometries and scattering probabilities make this choice preferable to a vanadium spectrum.

\section{Theophylline}

First spectra were taken at the BSS instrument of FZJ with both, centered and offset configurations of the instrument, and have shown a clear tunneling band. With the better energy resolution of SPHERES at FRM2, Munich, the broad band is resolved as a doublet (Fig. 6). Since the two subbands must have equal intensities according to the crystal 


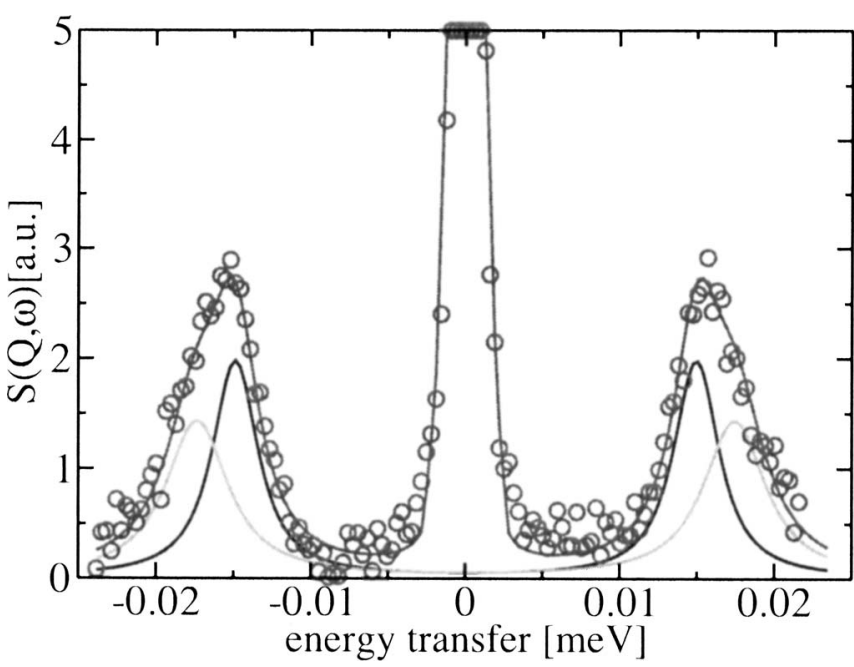

FIG. 6. Tunneling spectrum of theophylline $(\bigcirc \bigcirc \bigcirc)$. Sample temperature $T=2.6 \mathrm{~K}$. Spectrometer is SPHERES of JCNS, Munich. Wavelength $\lambda$ $=6.27 \AA$. Average momentum transfer $Q=1.5 \AA^{-1}$. Solid lines: Fit with a doublet tunneling band and a resolution function. The maximum of the elastic line is 320 .

structure, they come out to have different widths. Such a theory function is convoluted with the measured resolution function (Fig. 6, solid line). The fit yields tunneling bands at 15.1 and $17.5 \mu \mathrm{eV}$ and linewidths of 1.7 and $2.8 \mu \mathrm{eV}$, respectively. After all corrections the relative inelastic intensity agrees with a scattering function [Eq. (3)] and parameters $N=N^{\prime}=2$ and $M=2$. This means that the tunneling peaks represent all methyl groups. The crystal structure at $T=3 \mathrm{~K}$, thus, is likely the same as at room temperature with a single molecule in the irreducible unit cell. In agreement with this analysis, no further tunneling transition could be observed up to the free rotor energy in a spectrum measured at NEAT of HMI, Berlin. The finite linewidths of the tunnel peaks may be a sign of $\operatorname{disorder}^{23}$ or of coupling between methyl groups. $^{24}$

The temperature dependence of the tunneling bands is difficult to analyze reliably due to the overlapping lines and their intrinsic low temperature widths. If we describe the two bands by a single one, this band shifts toward the elastic line and broadens with sample temperature as qualitatively expected from theory ${ }^{25}$ for a quantum rotor coupled to a phonon bath. For the outlined difficulties we do not try a more quantitative analysis.

\section{Theobromine}

The elastic lines of raw spectra of theobromine are systematically broader as the resolution. This suggests the presence of an unresolved tunneling transition. Due to correlations of intensity and position of the unresolved Lorentzian, it is close to impossible to leave all parameters of the band free. The restriction in our fit of the data of Fig. 7 is twofold. At first, all curves are resolution functions. Secondly, since the fixed-window curve has shown that only $50 \%$ of the methyl groups are weakly hindered, the intensity of the tunneling band is varied together with the elastic intensity in the ratio found for theophylline. With the mentioned conditions a tunnel splitting of $(0.3 \pm 0.2) \mu \mathrm{eV}$ is determined. The large

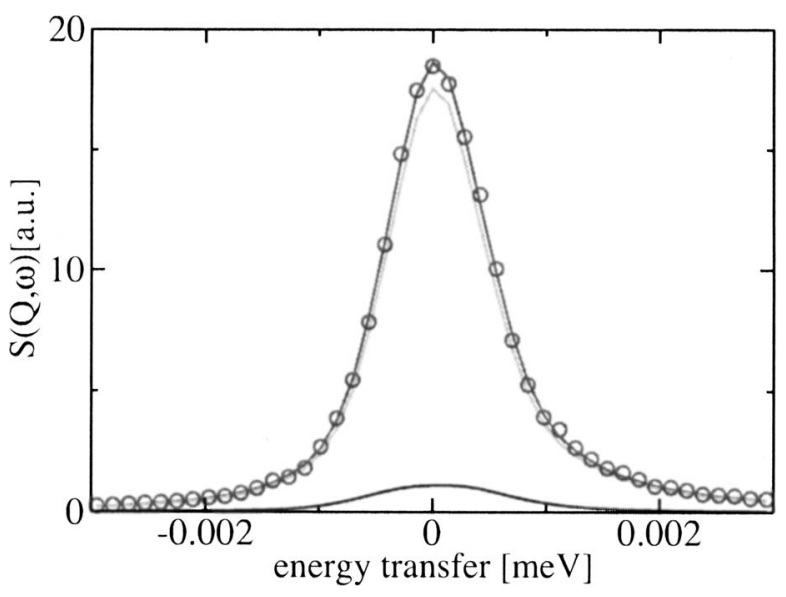

FIG. 7. Tunneling spectrum of theobromine $(\bigcirc \bigcirc \bigcirc)$. Sample temperature $T=4.5 \mathrm{~K}$. Spectrometer is BSS of FZJ, Jülich. Wavelength $\lambda=6.27 \AA$. Average momentum transfer $Q=1.5 \AA^{-1}$. Solid lines: Fit by an elastic resolution function and a tunneling doublet. Tunneling bands have 0.04 times the elastic intensity.

error bar is due to a strong dependence of the fit parameters, e.g., on the background subtraction. Even if we leave the intensity free, a pure resolution function cannot describe the spectrum but the fit finds a little more tunneling intensity at a lower energy transfer of $\sim 0.16 \mu \mathrm{eV}$. This clearly confirms the presence of the tunneling band.

\section{Caffeine}

In the orientationally disordered crystal, ${ }^{9,10}$ tunneling transitions do not form sharp bands. A first fingerprint of disorder is the broadened temperature interval on which elastic intensity gets lost in the fixed-window scan (Fig. 5). The onset of this intensity loss at very low temperatures further means that some rotational potentials are weak such that the corresponding fraction of tunneling systems should be observable as a wing of the elastic line in a high resolution spectrum as in some polymeric glassy materials. ${ }^{23}$ To emphasize this observation the elastic line in Fig. 8 is suppressed by a factor of $\sim 10$. At this scale, the three solid lines de-

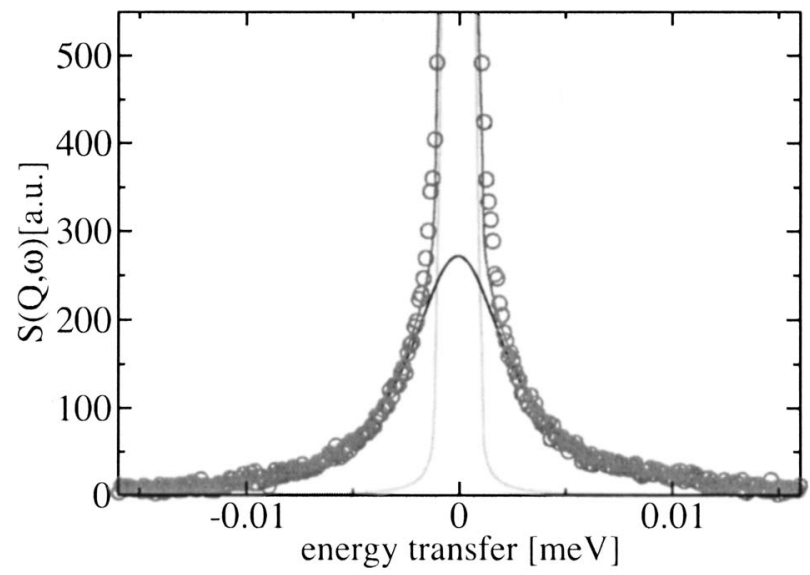

FIG. 8. High resolution spectrum of caffeine (○००). Sample temperature $T=2.55 \mathrm{~K}$. Spectrometer is SPHERES of JCNS at FRM2, Munich. Wavelength $\lambda=6.27 \AA$ A . Average momentum transfer $Q=1.5 \AA^{-1}$. Solid lines: Fit by a sum of a resolution function and a Lorentzian. For clarity, a resolution function is shown too. The elastic line is suppressed by a factor of 10 . 
TABLE II. Characteristic mode energies of theophylline, theobromine, caffeine, and paraxanthine, as derived from Figs. 9-15. The tunnel splitting of caffeine is taken as half of the width of the inelastic Lorentzian. Sample temperature $T=2.4-20 \mathrm{~K}$. A tentative assignment of methyl librations is shown in parentheses.

\begin{tabular}{lccc}
\hline \hline Theophylline & Theobromine & Caffeine & Paraxanthine \\
\hline 0.0145 & 0.0003 & $(0.0013)$ & \\
$3.13(L)$ & & & \\
6.46 & 5.55 & 6.4 & 6.59 \\
7.92 & 8.87 & & 8.90 \\
11.46 & & & 12.06 \\
& 14.14 & $14.6(L)$ & $15.03(L)$ \\
$14.8(L 2)$ & $15.53(L)$ & & 16.70 \\
& $16.63(L)$ & 18.7 & $19.35(L)$ \\
$19.5(L 2)$ & 18.67 & & 22.9 \\
& $21.2(L)$ & & 27.9 \\
23.9 & $22.7(L)$ & 28.0 & 34.0 \\
34.2 & 29.3 & 35.9 & 44.0 \\
37.8 & & 39.3 & 46.5 \\
44.5 & 37.2 & 46.7 & \\
49.0 & 43.6 & 69.6 & \\
& 48.7 & 76.7 & \\
& & & \\
\hline \hline
\end{tabular}

scribe the elastic resolution function, a Lorentzian to model the distribution of tunneling intensity, and the sum of both. The broad Lorentzian thus contains both, the up-and downscattered tunneling modes. Linewidths and intensities are obtained by convoluting the theoretical spectrum with the measured resolution function. The intensities of the elastic line, the inelastic line, and their width were free parameters of the fit. The fraction of rotor systems contributing to the inelastic wing are estimated from the relative Lorentzian intensity $I_{\text {incl }} / I_{\mathrm{el}}=0.28$. This is in very good agreement with a value of 0.30 (sum of both inelastic bands) calculated from Eq. (3) for a model with $2 / 3$ of the rotors performing tunneling proposed from the intensity loss in the fixed-window scan (column 4 of Table I). A mean tunneling frequency $\hbar \omega_{t} \sim \Gamma / 2$ can be guessed from the width of the Lorentzian band and is used below to derive an average rotational potential.

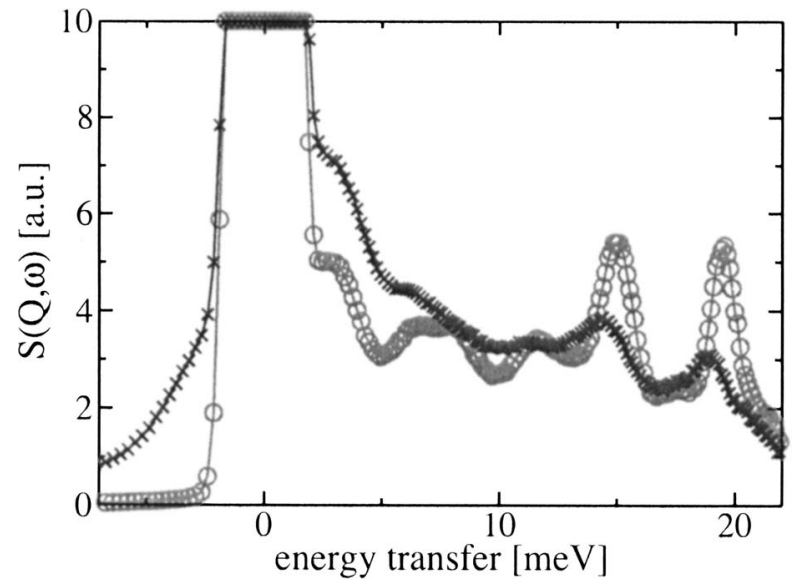

FIG. 9. DOS of theophylline. Sample temperatures $T=3.0(\bigcirc)$ and $60 \mathrm{~K}$ (xxx). Spectrometer is SV29, $\lambda=1.76 \AA$.

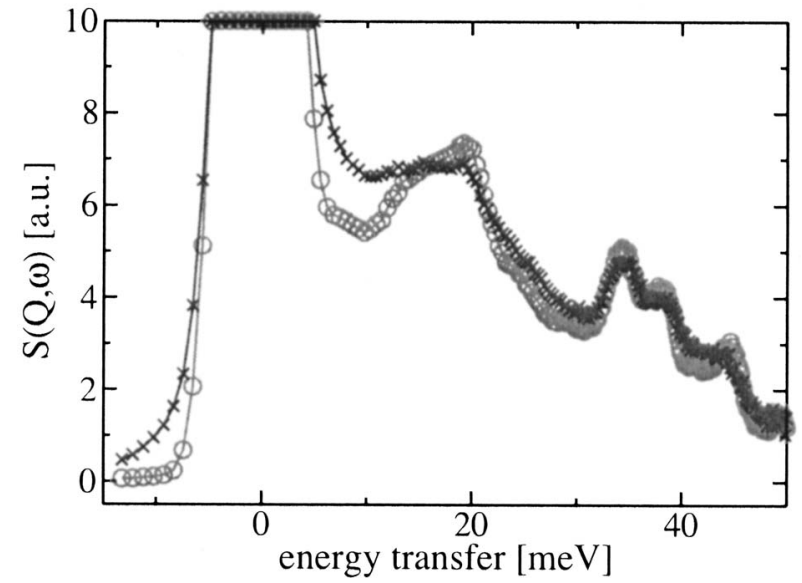

FIG. 10. DOS of theophylline. Sample temperatures $T=3.0(\bigcirc)$ and $60 \mathrm{~K}$ (xxx). Spectrometer is SV29, $\lambda=1.17 \AA$.

\section{VIBRATIONAL DENSITY OF STATES}

A Raman study combined with ab initio modeling of caffeine ${ }^{26}$ shows that internal modes of the isolated molecule have energies above $25 \mathrm{meV}$. The lowest mode at $28 \mathrm{meV}$ in the cited study was assigned to methyl liberation. The actual experiment uses polycrystals of all samples. The energies and tentative assignments of lattice modes are shown in Table II. The identification of methyl librations is based on the properties of the incoherent neutron scattering function from phonons. ${ }^{27}$ At first, the large scattering cross section of the hydrogen involved together with the large amplitude of librational modes make liberations especially strong among all other lattice modes. (However, the presence of dispersion may smear out this intensity over a large energy range. ${ }^{28}$ ) Next, their anharmonicity makes librational modes especially sensitive to temperature with a corresponding strong damping.

Librational modes in the solid state (Figs. 9-15) look significantly softened compared to the isolated molecule. Thus, density functional theory calculations on isolated molecules do not contribute to a quantitative understanding of lattice modes in the solid.

While the transition energies of theobromine and parax-

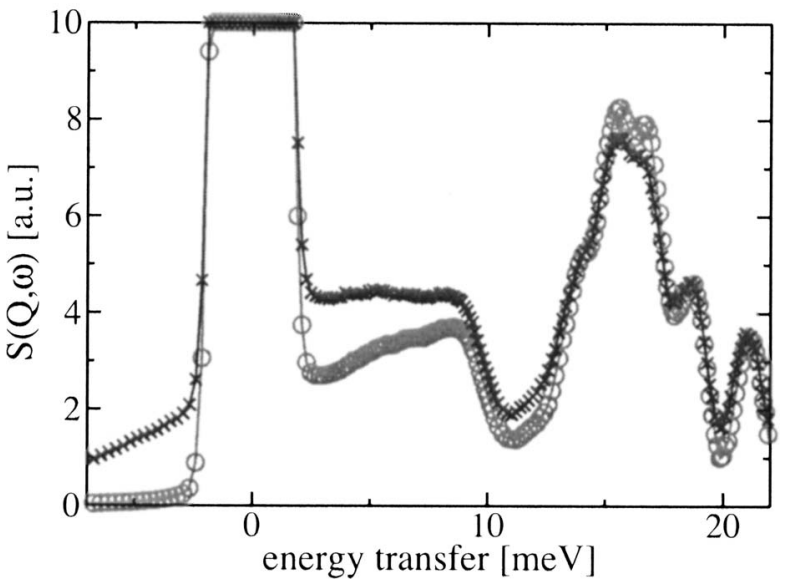

FIG. 11. DOS of theobromine. Sample temperatures $T=3.0(\bigcirc)$ and $60 \mathrm{~K}$ (xxx). Spectrometer is SV29, $\lambda=1.76 \AA$. 


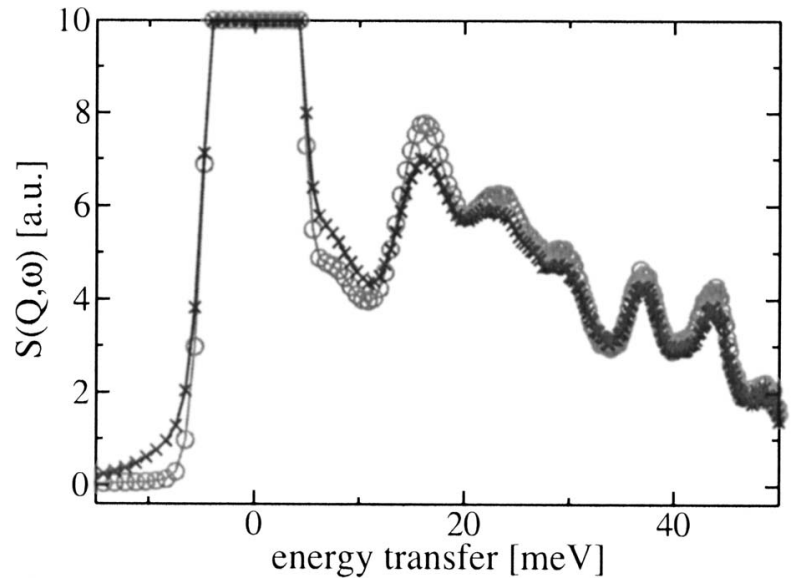

FIG. 12. DOS of theobromine. Sample temperatures $T=3.0(\bigcirc)$ and $60 \mathrm{~K}$ (xxx). Spectrometer is SV29, $\lambda=1.17 \AA$.

anthine are almost independent of temperature, those of theophylline below $20 \mathrm{meV}$ soften by $\sim 3 \%$ at a sample temperature $T=60 \mathrm{~K}$. The mode at $7.92 \mathrm{meV}$ even seems to disappear (Fig. 9). The reason of such unusual changes could be a phase transition whose study would require further experiments. Only theophylline shows a strong exceptionally low energy mode at $3.13 \mathrm{meV}$.

In the case of caffeine, well-defined librational modes do not exist because of orientational disorder. The strongest and broad band at about $15 \mathrm{meV}$ must represent the average librational energy. A combination with other, also error affected, rotational parameters (mean tunnel splitting, mean rotational barrier) is hoped to lead us to a consistent description by a common rotational potential. This is the task of the following section.

\section{A. Methyl rotational potentials}

The Schrödinger equation (2) links all rotational excitations of a methyl group by the rotational potential $V(\varphi) . E_{a}$ from the fixed-window scan, the characteristic librational energies in the phonon spectra, and the methyl rotational tunnel splittings must now be represented consistently by potential parameters $V_{3}$ and $V_{6}$ characterizing the shape and strength

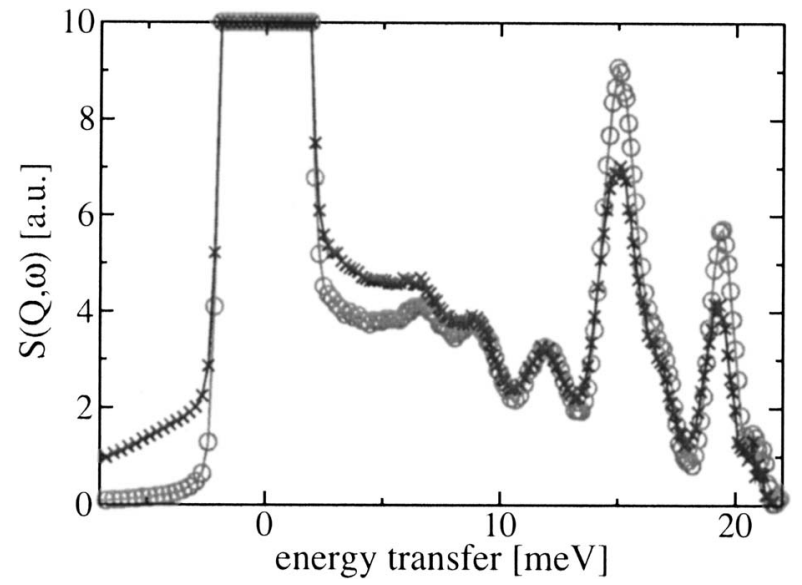

FIG. 13. DOS of 1,7-dimethylaxanthine. Sample temperatures $T=3.0(\bigcirc)$ and $60 \mathrm{~K}(\mathrm{xxx})$. Spectrometer is SV29, $\lambda=1.76 \AA$.

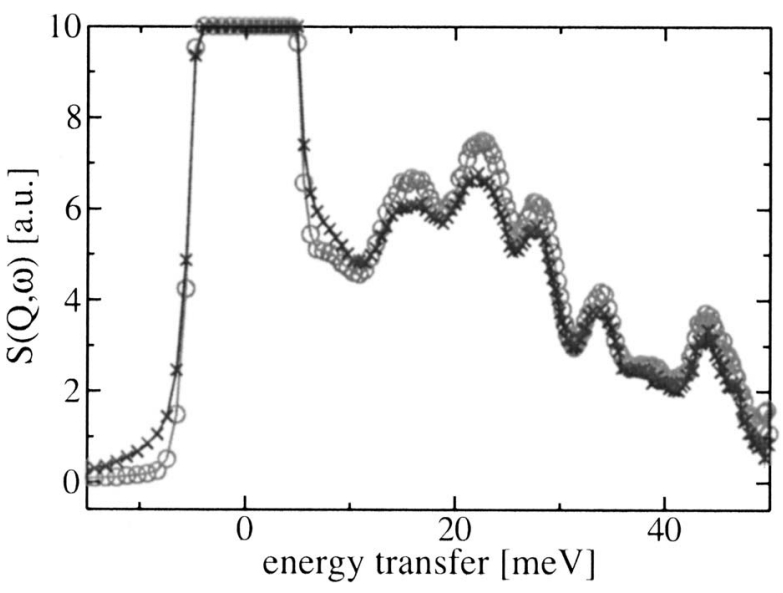

FIG. 14. DOS of 1,7-dimethylxanthine. Sample temperatures $T=3.0(\bigcirc)$ and $60 \mathrm{~K}(\mathrm{xxx})$. Spectrometer is SV29, $\lambda=1.17 \AA$.

of $V(\varphi)$. Thereby, one has to keep in mind that only in the classical limit of $T_{1 / 2} \geqslant 50 \mathrm{~K}$, an activation energy $E_{a}$ represents the distance of a rotational barrier from the groundstate level. Since there is no symmetry connecting the methyl groups within a molecule, there must be at least as many different species as methyl groups in the formula unit. At room temperature this is the case of theobromine and theophylline. ${ }^{5,7,9}$ In the cases of paraxanthine with a cell doubling and of caffeine with its glassy orientational structure, our data allow the application of simplified models only.

Table III shows the results of this procedure. On the left hand side are the input parameters, in the middle are the potential parameters matching these observables, and on the right hand side, there are some calculated quantities to be discussed. If eigenenergies are not shown in the last column, they match the input parameters. In the case of caffeine, all observables are broad and overlapping due to orientational disorder. Despite of large error bars of the observables, a mean rotational potential of the glassy system can be found, which describes the system very well. If we split in mind the inelastic Lorentzian into two halves describing the energy gain and loss transitions, we can estimate an average tunneling energy $\hbar \omega_{t} \sim \Gamma / 2$, which is completely consistent with the calculated $\hbar \omega_{t}=1.3 \mu \mathrm{eV}$. To derive a reliable width of

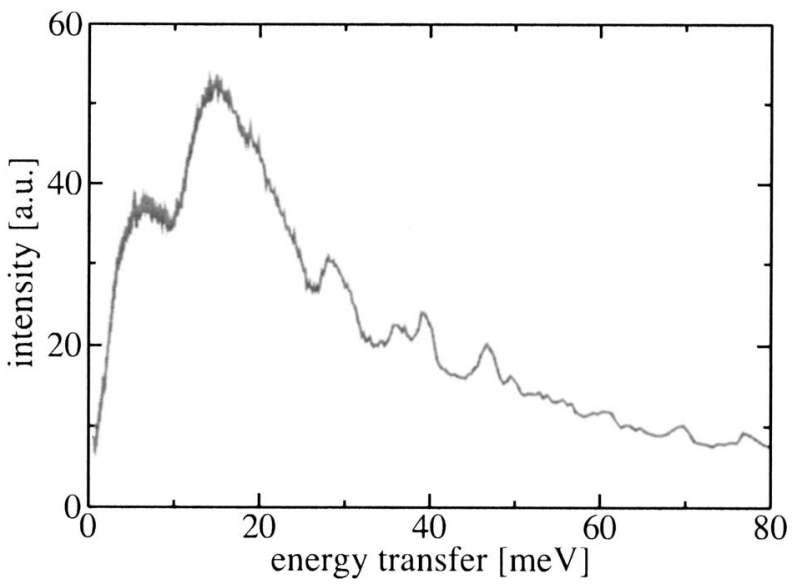

FIG. 15. DOS of caffeine. Sample temperature $T=20 \mathrm{~K}$. Spectrometer is NERA of JINR Dubna. 
TABLE III. Rotational potential parameters $V_{3}$ and $V_{6}$ of methylxanthines adjusted to describe best the measured quantities shown in columns $2-4$. The potential delivers calculated values shown in the last column.

\begin{tabular}{|c|c|c|c|c|c|c|}
\hline Sample & $\begin{array}{c}\hbar \omega_{t} \\
(\mu \mathrm{eV})\end{array}$ & $\begin{array}{c}E_{01} \\
(\mathrm{meV})\end{array}$ & $\begin{array}{c}E_{a} \\
(\mathrm{meV})\end{array}$ & $\begin{array}{c}V_{3} \\
(\mathrm{meV})\end{array}$ & $\begin{array}{c}V_{6} \\
(\mathrm{meV})\end{array}$ & Calc. \\
\hline \multirow[t]{2}{*}{ Theophylline } & 15.1 & 3.13 & & 3.45 & 52 & $E_{a}=38 \mathrm{meV}$ \\
\hline & 17.5 & 3.13 & & 3.45 & 50 & $E_{n}=35 \mathrm{meV}$ \\
\hline \multirow[t]{2}{*}{ Theobromine } & 0.3 & 16.1 & 53 & 66.5 & -6 & $\begin{aligned} \hbar \omega_{t} & =0.37 \mu \mathrm{eV} \\
E_{n} & =58 \mathrm{meV}\end{aligned}$ \\
\hline & & 22 & 88 & 99.5 & -2 & $\hbar \omega_{t}=0.02 \mu \mathrm{eV}$ \\
\hline Caffeine & $(\Gamma=2.7 \mu \mathrm{eV})$ & 14.6 & 43 & 50.5 & -2.7 & $\hbar \omega_{t}=1.3 \mu \mathrm{eV}$ \\
\hline \multirow[t]{2}{*}{ Paraxanthine } & & 15.0 & 55 & 62.0 & -7 & $\hbar \omega_{t}=0.6 \mu \mathrm{eV}$ \\
\hline & & 19.4 & 72 & 81.0 & -3 & $\hbar \omega_{t}=0.09 \mu \mathrm{eV}$ \\
\hline
\end{tabular}

the potential distribution function, however, would require a more detailed analysis as it was developed for glassy polymers. $^{22}$

In the case of paraxanthine, the inhomogeneously broadened step in the fixed-window scan contains the contributions from all four inequivalent methyl groups of the unit cell. Not to overinterpret the smooth intensity decay, we use only two inequivalent species of methyl groups in the model analysis and postulate equal step heights. The two different mean activation energies and the corresponding two rotational potentials determine the energy regime where corresponding bands of the four different methyl species can be expected. A too large calculated tunnel splitting, which was not observed, shows the limits of such simplifying analysis.

In the case of theobromine, the two inequivalent methyl groups have such different potentials that the steps in the fixed-window scan are clearly separated. In a first attempt, the two activation energies and the librations were used to determine the potential parameters. For the resulting rather weak potential a finite small tunnel splitting was calculated which should be observable. This has led to a reanalysis of the tunnel spectrum and the detection of a tunnel spitting of $0.3 \mu \mathrm{eV}$. Therewith, theobromine is the only material where three observables for one methyl group are available. The derived two-parameter potential (Table III) is a compromise to describe best all three observables simultaneously. The error bars of two of the input parameters, the small and weak tunnel splitting and the activation energy, affect these potential parameters with significant error bars too.

In all three materials discussed so far, the symmetry of the rotational potentials is dominated by the threefold symmetry of the methyl group with large values of $V_{3}$. In the case of theophylline, we have the clearest spectral information in the form of the resolved tunnel bands. From the three sharp equally strong bands of the density of states (DOS), only the combination with the lowest strong band at $3.1 \mathrm{meV}$ allows descriptions by the Mathieu equation. The fitted potential is dominantly sixfold, in contrast to the cases of theobromine, paraxanthine, and caffeine. In this potential, the second librational doublet state appears at rather high energy of around $26 \mathrm{meV}$ due to the strong curvature of the potential at its minimum. This calculated energy has to be compared with the two other measured strong transitions at 14.8 and $19.5 \mathrm{meV}$. These modes may be tentatively assigned to second harmonic librations. The discrepancy with the calculated energies lies most likely in the unusual symmetry of the rotational potential. If the fundamental symmetry is close to six, one is not allowed to neglect terms of the next higher orders of nine and twelve. This is the same argument used for the standard situation that the term $V_{6}$ plays an important role if the fundamental symmetry is threefold. A crystallographic study of the theophylline monohydrate ${ }^{29}$ has shown that the protons of the methyl group at the 3-position of the ring in this related material are indeed distributed on a hexagon. The strongly sixfold symmetry of the rotational potential favors the overlap of the molecular wave function and leads to a large tunnel splitting - as observed in anhydrous theophylline. The unusual potential may also be the reason for the unusually strong softening of some bands of the density of states of this compound with temperature. The activation energy of $38 \mu \mathrm{eV}$ calculated with the proposed potential should and does not coincide with the activation energy obtained from the fixed-window scan (Table I), since the evolution of the tunneling spectrum with temperature ${ }^{25}$ follows different laws from a classical methyl rotor. The most careful study of tunneling spectra in the regime between quantum and classical behaviors shows ${ }^{22}$ that the line broadening bends always away from the classical Arrhenius behavior to higher values while entering the quantum regime. An estimate based on a single point in this temperature regime and a constant prefactor of the Arrhenius function must, therefore, yield a too low activation energy.

\section{CONCLUSION}

The dimethylxanthines and trimethylxanthine were studied by neutron spectroscopy up to energy transfers of $100 \mathrm{meV}$ at energy resolutions ranging from $0.7 \mu \mathrm{eV}$ to some meV. For all materials, the loss of elastic intensity with increasing temperature observed in backscattering elastic fixed-window scans shows a momentum transfer dependence consistent with methyl group rotation. All involved methyl rotational potentials are weak with activation energies between 35 and $90 \mathrm{meV}$. In all materials, the number of different dynamical components and their intensities are consistent with the number of inequivalent methyl groups determined 
by diffraction at room temperature. In some cases, the rotational potentials are too similar to be distinguished by the integral fixed-window technique. In theophylline, the tunneling bands of the two inequivalent methyl groups are almost degenerate by chance. In this material, a dominantly sixfold rotational potential can consistently model a low lying librational mode and a large tunnel splitting. Spectra from caffeine display very broad bands, especially in the tunneling regime. They characterize a distribution of methyl rotational potentials due to the presence of an orientational glass state. The potential distribution function is such that about $\sim 70 \%$ of the methyl groups have tunnel splittings larger than the instrumental resolution of $0.7 \mu \mathrm{eV}$. Parameters of an average potential are derived. The four inequivalent methyl groups of paraxanthine can only be characterized by two types of rotational potentials due to a likely accidental similarity of each of two rotor species. The clearest case is that of theobromine with two clearly different rotational potentials where even the integral fixed-window technique can identify two activation energies.

A convincing interpretation of the lattice dynamics of the methylxanthines requires mathematical modeling. ${ }^{30,31}$ This is only possible on the basis of high quality low temperature crystal structures. Such calculations would also shed new light on the effect of hydrogen bonds.

\section{ACKNOWLEDGMENTS}

We thank Margerita Russina and the HMI for a test experiment showing that there are no high energy tunneling modes in theophylline beyond $30 \mu \mathrm{eV}$.

${ }^{1}$ M. Johnson, M. Prager, H. Grimm, M. A. Neumann, G. J. Kearley, and C. C. Wilson, Chem. Phys. 244, 49 (1999).

${ }^{2}$ A. Detken, P. Schiebel, M. R. Johnson, H. Zimmermann, and U. Haeberlen, Chem. Phys. 238, 301 (1998).

${ }^{3}$ C. E. Müller, D. Shi, M. Manning, and J. W. Daly, J. Med. Chem. 36, 3341 (1993)

${ }^{4}$ H. Moustafa, S. H. Shalaby, K. M. El-Sawy, and R. Hilal, Spectrochim. Acta, Part A 58, 2013 (2002).

${ }^{5}$ Y. Ebisuzaki, P. D. Boyle, and J. A. Smith, Acta Crystallogr., Sect. C: Cryst. Struct. Commun. 53, 777 (1997).
${ }^{6}$ E. D. L. Smith, R. B. Hammond, M. J. Jones, K. J. Roberts, J. B. O. Mitchell, S. L. Price, R. K. Harris, D. C. Appearly, J. C. Cherryman, and R. Docherty, J. Phys. Chem. B 105, 5818 (2001).

${ }^{7}$ K. A. Ford, Y. Ebisuzaki, and P. D. Boyle, Acta Crystallogr., Sect. C: Cryst. Struct. Commun. C54, 1980 (1998)

${ }^{8}$ L. Carlucci and A. Gavezzotti, Chem.-Eur. J. 11, 271 (2005).

${ }^{9}$ D. J. Sutor, Acta Crystallogr. 11, 453 (1958).

${ }^{10}$ P. Derollez, N. T. Correira, F. Dante, F. Capet, F. Affouard, J. Lefebre, and M. Descamps, Acta Crystallogr., Sect. B: Struct. Sci. B61, 329 (2005).

${ }^{11}$ M. Ghosh, A. K. Basak, S. K. Mazumdar, and B. Sheldrick, Acta Crystallogr., Sect. C: Cryst. Struct. Commun. C47, 577 (1991).

${ }^{12}$ A. del Rio, A. Boucekkine, and J. Meinnel, J. Comput. Chem. 24, 2093 (2003).

${ }^{13}$ W. Press, in Single Particle Rotations in Molecular Crystals, Springer Tracts in Modern Physics Vol. 81 (Springer, Berlin, 1981).

${ }^{14}$ M. Prager and A. Heidemann, Chem. Rev. (Washington, D.C.) 97, 2933 (1997).

${ }^{15}$ The equipartition principle for free $\mathrm{CH}_{3}$ rotation at room temperature, $(1 / 2) \Theta \omega_{0}^{2}=(1 / 2) k T$, yields $\omega_{0} / 2 \pi \sim 1 \mathrm{THz}$.

${ }^{16}$ T. Springer, Dynamics of Solids and Liquids by Neutron Scattering, Topics in Current Physics Vol. 3, edited by S. W. Lovesey and T. Springer (Springer, Berlin, 1977), p. 284.

${ }^{17}$ See http://www.fz-juelich.de/iff/Institute/ins/Broschuere_NSE/ for SV29.

${ }^{18}$ See http://www.jens.info/jcns_sphere for SPHERES.

${ }^{19}$ I. Natkaniec, S. I. Bragin, J. Brankowski, and J. Mayer, in Proceedings of ICANS-XIII, Abingdon, 1993, RAL Report No. 94-025, Vol. 1, P. 89.

${ }^{20}$ H.-H. Grapengeter, B. Alefeld, and R. Kosfeld, Colloid Polym. Sci. 265, 226 (1987).

${ }^{21}$ A. Heidemann, M. Prager, and M. Monkenbusch, Z. Phys. B 76, 77 (1989).

${ }^{22}$ A. Würger and A. Heidemann, Z. Phys. B 80, 113 (1990).

${ }^{23}$ J. Colmenero, R. Mukhopadhyay, A. Alegria, and B. Frick, Phys. Rev. Lett. 80, 2350 (1998).

${ }^{24}$ G. Voll, Physica B 202, 239 (1994).

${ }^{25}$ A. Hewson, J. Phys. C 15, 3841 (1982); 15, 3855 (1982)..

${ }^{26}$ I.-E. Pavel, Ph.D. thesis, University Würzburg, 2003.

${ }^{27}$ S. Lovesey, Dynamics of Solids and Liquids by Neutron Scattering, Topics in Current Physics, edited by S. W. Lovesey and T. Springer (Springer, Berlin, 1977), Vol. 3, p. 29.

${ }^{28}$ O. Kirstein, M. Prager, M. R. Johnson, and S. F. Parker, J. Chem. Phys. 117, 1313 (2002).

${ }^{29}$ C. Sun, D. Zhou, D. J. W. Grant, and V. G. Young, Jr., Acta Crystallogr., Sect. E: Struct. Rep. Online E58, 8368 (2002).

${ }^{30}$ M. Prager, W. I. F. David, and R. M. Ibberson, J. Chem. Phys. 95, 2473 (1991).

${ }^{31}$ M. R. Johnson and G. J. Kearley, Annu. Rev. Phys. Chem. 51, 297 (2000). 